\title{
Disambiguation of Fingerprint Ridge Flow Direction-Two Approaches
}

\author{
Robert O. Hastings \\ School of Computer Science and Software Engineering, \\ The University of Western Australia, Australia \\ http://www.csse.uwa.edu.au/ bobh
}

\begin{abstract}
One of the challenges to be overcome in automated fingerprint matching is the construction of a ridge pattern representation that encodes all the relevant information while discarding unwanted detail. Research published recently has shown how this might be achieved by representing the ridges and valleys as a periodic wave. However, deriving such a representation requires assigning a consistent unambiguous direction field to the ridge flow, a task complicated by the presence of singular points in the flow pattern. This disambiguation problem appears to have received very little attention.

We discuss two approaches to this problem - one involving construction of branch cuts, the other using a divide-and-conquer approach, and show how either of these techniques can be used to obtain a consistent flow direction map, which then enables the construction of a phase based representation of the ridge pattern.
\end{abstract}

\section{Introduction}

A goal that has until recently eluded researchers is the representation of a fingerprint in a form that encodes only the information relevant to the task of fingerprint matching, i.e. the details of the ridge pattern, while omitting extraneous detail.

Level 1 detail, which refers to the ridge flow pattern and forms the basis of the Galton-Henry classification of fingerprints into arch patterns, loops, whorls etc., (Maltoni et al., 2003, p 174) is encapsulated in the ridge orientation field. Level 2 detail, which refers to details of the ridges themselves, especially instances where ridges bifurcate or terminate, is the primary tool of fingerprint based identification, and it is not so obvious how best to represent this. A popular approach has been to define ridges as continuous lines defining the ridge axes. For example, Ratha et al. (1995) convert the grey-scale image into a binary image, then thin the ridges to construct a "skeleton ridge map" which they then represent by a set of chain codes. Shi and Govindaraju (2006) employ chain codes to represent the ridge edges rather than the axes of the ridges - that is, the ridges are allowed to have a finite width. This avoids the need for a thinning step, but still requires that the image be binarised. 
Some problems with the skeleton image representation are:

1. The output of thinning is critically dependent on the chosen value of the binarisation threshold, and is also highly sensitive to noise.

2. It is not immediately clear how one might quickly determine the degree of similarity of two given chain codes.

An alternative is to represent the ridges via a scalar field, the value at each point specifying where that point is relative to the local ridge and valley axes. The phase representation, discussed in the next section, is a way to achieve this.

\section{Representation of the Ridges as a Periodic Wave}

Except near core and delta points, the ridges resemble the peaks or troughs of a periodic wave train. This suggests that the pattern might be modeled using, say, the cosine of some smoothly varying phase quantity. Two fingerprint segments could then be compared directly with one another by taking the point-wise correlation of the cosine values. There are two major difficulties with this approach:

1. Any wave model must somehow incorporate the Level 2 detail (minutiae), meaning that the wave phase must be discontinuous at these points.

Recently published research describes a phase representation in which the minutiae appear as spiral points in the phase field (Sect. 2.1).

2. Deriving a phase field implies the assignment of a direction to the wave. Whilst it is relatively easy to obtain the ridge orientation, disambiguating this into a consistent flow field is a non-trivial task. The challenge of disambiguation is the main theme of this paper, and is discussed in Sect. 3.

\subsection{The Larkin and Fletcher Phase Representation}

Larkin and Fletcher (2007) propose a finger ridge pattern representation based on phase, with the grey-scale intensity taking the form:

$$
I(x, y)-a(x, y)=b(x, y) \cos [\psi(x, y)]+n(x, y),
$$

where $I$ is image intensity at each point, $a$ is the offset, or "DC component", $b$ is the wave amplitude, $\psi$ is the phase term and $n$ is a noise term. The task is to determine the parameters $a, b$ and $\psi$; this is termed demodulation.

After first removing the offset term $a(x, y)$ by estimating this as the mid-value of a localised histogram, they define a demodulation operator $\$$ and apply this to the remainder. They show that, neglecting the noise term:

$$
\$\{b(x, y) \cos [\psi(x, y)]\} \approx-i \exp [i \beta(x, y)] b(x, y) \sin [\psi(x, y)],
$$

where $\beta$ is the direction of the wave normal. Comparison of (11) and (2) shows that the right hand sides are in the ratio:

$$
-i \exp [i \beta(x, y)] \tan [\psi(x, y)]
$$

so that provided we know $\beta$ we can use (3) to determine the phase term $\psi$. 
By the Helmholtz Decomposition Theorem (Joseph, 2006), $\psi$ can be decomposed into a continuous component $\psi_{c}$ and a spiral component $\psi_{s}$, where $\psi_{s}$ is generated by summing a countable number of spiral phase functions centred on $n$ points and defined as 1 :

$$
\psi_{s}(x, y)=\sum_{i} p_{i} \arctan \left(\frac{y-y_{i}}{x-x_{i}}\right) .
$$

The points $\left\{\left(x_{i}, y_{i}\right)\right\}$ are the locations of spirals in the phase field; each has an associated "polarity" $p_{i}= \pm 1$. These points can be located using the Poincaré Index, defined as the total rotation of the phase vector when traversing a closed curve surrounding any point (Maltoni, Maio et al., 2003, p 97). This quantity is equal to $+2 \pi$ at a positive phase vortex, $-2 \pi$ at a negative vortex and zero everywhere else. The residual phase component $\psi_{c}=\psi-\psi_{s}$ contains no singular points, and can therefore be unwrapped to a continuous phase field.

Referring to (3), note that replacement of $\beta$ by $\beta+\pi$ implies a negation of $\psi$, so that, in order to derive a continuous $\psi$ field, we must disambiguate the ridge flow direction to obtain a continuous wave normal across the image.

\section{Disambiguating the Ridge Orientation}

Deriving the orientation field is comparatively straightforward. We use the methodology of Bazen and Gerez (2002), who compute orientation via Principal Component Analysis applied to the squared directional image gradients. The output from this analysis is an expression for the doubled angle $\phi=2 \theta$, with $\theta$ being the orientation. This reflects the fact that $\theta$ has an inherent ambiguity of $\pi$.

Inspection of the orientation field around a core or delta point (Fig. 1) reveals that, in tracing a closed curve around the point, the orientation vector rotates through an angle of $\pi$ in the case of a core, and through $-\pi$ for a delta. The Poincaré Index of $\phi$ is therefore $2 \pi$ at a core, $-2 \pi$ at a delta, and zero elsewhere.

Larkin and Fletcher note in passing that their technique requires that the orientation field be unwrapped to a direction field, but Fig. 2 illustrates the difficulty inherent in determining a consistent direction field. The difficulty arises from the presence of a singular point (in this case a core). This unwrapping task appears to received scant attention to date, perhaps because there has been no clear incentive for doing so prior to the publication of the ridge phase model. Sherlock and Monro (1993) discuss the unwrapping of the orientation field (which they term the "direction field"), but this is a different and much simpler task, because the orientation, expressed as the doubled angle $\phi$, contains only singular points rather than lines of discontinuity.

\footnotetext{
${ }^{1}$ In this paper the $\arctan$ function is understood to be implemented via $\arctan (y / x)=$ atan2 $(y, x)$, where the atan2 function returns a single angle in the correct quadrant determined by the signs of the two input arguments.
} 


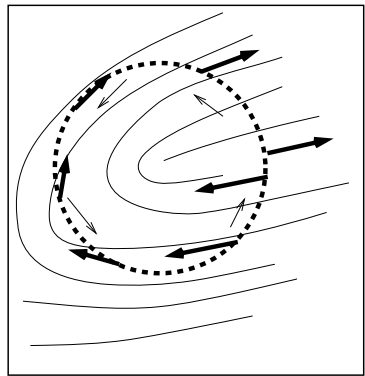

(a) Core

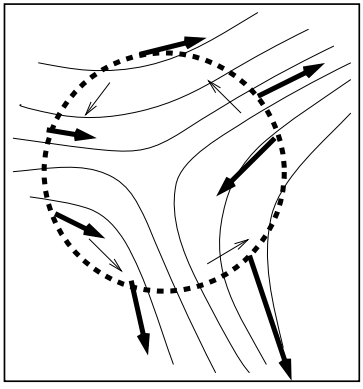

(b) Delta

Fig. 1. Closed loop surrounding a singular point, showing the orientation vector (dark arrows) at various points around the curve

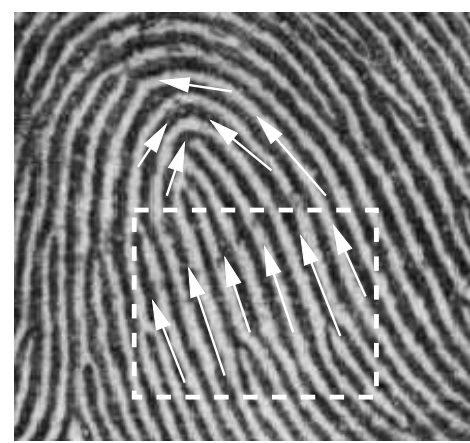

(a) An unsuccessful attempt to assign a consistent flow direction

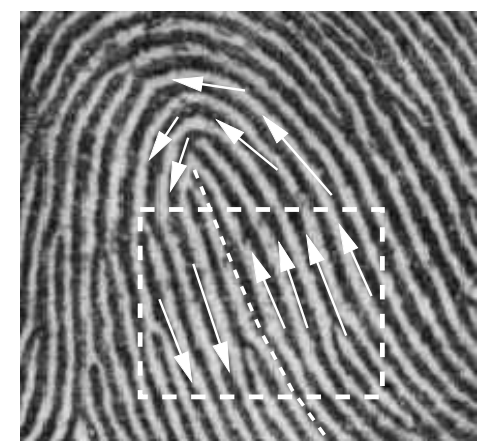

(b) Flow direction correctly assigned with consistency across the image

Fig. 2. A flow direction that is consistent within a region (dashed rectangle) cannot always be extended to the rest of the image without causing an inconsistency (a). Reversing the direction over part of the image resolves this inconsistency (b).

We discuss here two possible approaches to circumventing this difficulty:

1. Construct a flow pattern in which regions of oppositely directed flow are separated by branch cuts, as illustrated in Fig. 2(b).

2. Bypass the problem of singular points by subdividing the image into a number of sub-images. It is always possible to do this in such a way that no cores or deltas occur within any of the sub-images.

Both of these approaches were tried, and the methods and results are now described in detail. While the first approach is perhaps the more appealing, it does possess some shortcomings, as will be seen. 


\subsection{Disambiguation via Branch Cuts}

Examining Fig. 2(b), we see that we can draw a branch cut (sloping dashed line) down from the core point to mark the boundary between two oppositely directed flow fields. Our strategy is to trace these lines in the orientation field, define a branch cut direction field that exhibits the required discontinuity properties, and subtract this from the orientation field, leaving a continuous residual orientation field that can be unwrapped. The unwrapped field is then recombined with the branch cut field to give a final direction field that is continuous except along the branch cuts, where there is a discontinuity of $\pi$.

We define a dipole field $\phi_{d}$ on a line segment (illustrated in Fig. 3) as follows:

$$
\phi_{d}\left(x, y, x_{1}, y_{1}, x_{2}, y_{2}\right)=\arctan \left(\frac{y-y_{1}}{x-x_{1}}\right)-\arctan \left(\frac{y-y_{2}}{x-x_{2}}\right),
$$

where $\left(x_{1}, y_{1}\right)$ and $\left(x_{2}, y_{2}\right)$ are the start and end points of the line. If $\phi_{d}$ is defined to lie between $-\pi$ and $\pi$, this gives a discontinuity of $2 \pi$ only along the line itself (Fig. 3(a) . This is precisely what is required, except that we must later divide by 2 to give a discontinuity of $\pi$ rather than $2 \pi$. There is also a phase spiral at each end of the dipole (Fig. 3(b).

Branch cuts such as the one shown in Fig. 2(b) are constructed by commencing at a singular point and constructing a list of nodes $\left\{\left(x_{i}, y_{i}\right)\right\}$. The first node is the location of the singular point; each subsequent node is located by drawing a straight line segment from the previous node following the ridge orientation (which is already known). Further nodes are added to the list until the image

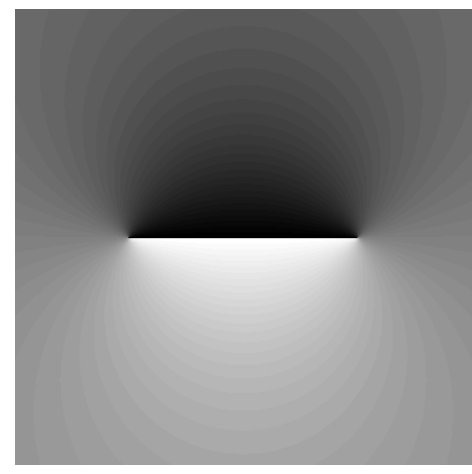

(a) Phase dipole field (grey scale).

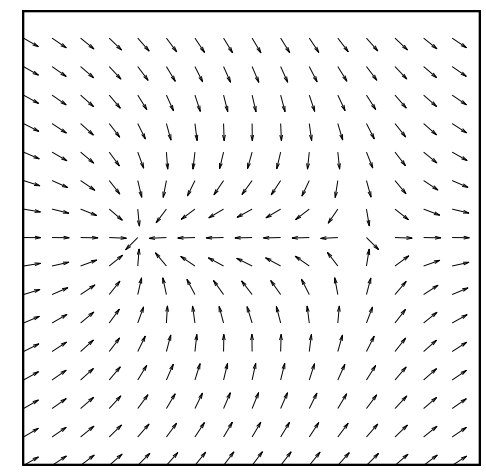

(b) Phase dipole field, shown in vector form.

Fig. 3. Phase field around a phase dipole. The positive end of the dipole is on the left, the negative on the right. Grey-scale values in (a) range from $-\pi$ (black) to $+\pi$ (white); direction values in (b) increase anticlockwise with zero towards the right. Note from (a) that the field is continuous everywhere except at the two poles and along the line between them. The linear discontinuity is not apparent in (b), because the directions of $\pi$ and $-\pi$ are equivalent. 
border is reached. Each core point is the source of one branch cut, while three branch cuts emanate from each delta point (see for example Fig.4(b)). A branch cut phase field $\phi_{b}$ is then defined for each individual branch cut:

$$
\phi_{b}(x, y)=\sum_{i=1}^{n-1} \phi_{d}\left(x, y, x_{i}, y_{i}, x_{i+1}, y_{i+1}\right) .
$$

Positive and negative dipole phase spirals cancel at each node except for the first and last nodes, leaving only a linear discontinuity of $2 \pi$ along each segment of the cut, plus a positive phase spiral at the start of the branch cut and a negative spiral at the end. In most cases the end node of a branch cut is outside the image so that it can be ignored (see however Sect. 4 where this is presented as one of the shortcomings of the branch cut based method of disambiguation).

Although $\Phi_{N}$ contains phase spirals at the same locations as $\phi$, the Poincaré Index does not have the correct value at the delta points, because the three branch cuts emanating from the point contribute a total of $3 \times 2 \pi=6 \pi$ to the Index, whereas for $\phi$ the value of the Index at a delta is $-2 \pi$. To correct this, we define an additional spiral field $\phi_{s}$ :

$$
\phi_{s}(x, y)=\sum_{i} \arctan \left(\frac{y-y_{i}}{x-x_{i}}\right),
$$

where $\left(x_{i}, y_{i}\right)$ is the location of the $i^{\text {th }}$ flow singularity and the summation is taken for all the core and delta points. The nett branch cut phase field $\Phi_{N}$ is now defined by:

$$
\Phi_{N}=2 \phi_{s}-\sum_{j} \phi_{b j}
$$

where the index $j$ refers to the $j^{\text {th }}$ branch cut. Inspection of (8) shows that:

- At a core, the Poincaré Index of $\Phi_{N}$ is $2 \times 2 \pi-2 \pi=2 \pi$.

- At a delta, the Poincaré Index of $\Phi_{N}$ is $2 \times 2 \pi-3 \times 2 \pi=-2 \pi$.

This matches the behaviour of $\phi$, meaning that $\Phi_{N}$ may now be subtracted from $\phi$ giving a residual field $\phi_{c}$ that can be unwrapped (giving $\phi_{c}^{\prime}$ ). A field $\phi^{\prime}$ is then generated by adding $\phi_{c}{ }^{\prime}$ back to $\phi_{s}$. Finally the result is halved. The resultant direction field $\theta^{\prime}$ now possesses the desired discontinuity properties, viz. a discontinuity of $\pi$ exists along each branch cut, and the Poincaré Index is $\pm \pi$ at a core or delta respectively.

\subsection{Disambiguation via "Divide and Conquer"}

The second method of obtaining a consistent flow direction does not require the construction of branch cuts but instead proceeds by progressively subdividing the image into a number of rectangular sub-images.

If the orientation field contains just one singular point $P$, we divide the image into four slightly overlapping rectangles 2 surrounding $P$. If there are two or

\footnotetext{
${ }^{2}$ The sub-images must be allowed to overlap slightly, because the Poincaré Index is obtained by taking differences, so that there is the risk of overlooking a minutia that lies close to the border of a sub-image.
} 
more singular points, partitioning is applied recursively by further subdividing any sub-image that contains a singular point. Each of the final sub-images is free of singular points, allowing a consistent flow direction field (and hence a wave normal direction field) to be assigned by directly unwrapping the orientation (Fig. 5(a) , though the directions may not match where the sub-images adjoin.

To avoid counting a minutia twice that occurs in a region of overlap, we set the minimum distance between minutiae to be $\bar{\lambda}$, the standard fingertip ridge spacing. Two minutiae closer than this distance are counted as one. To provide for generous overlap, the overlap distance is set at $3 \bar{\lambda}$. 3

\section{Results}

Ten-print images from the NIST14 and NIST27 Special Fingerprint Databases, supplied by the U.S. National Institute of Standards, formed the raw inputs for our work. In the results presented here, image regions identified as background are shown in dark grey or black. Segmentation of the image into foreground (discernible ridge pattern) and background (the remainder) is an important task, but is outside the scope of this paper.

\subsection{Flow Disambiguation Using Branch Cuts}

Figure 4 shows a portion of a typical input image and the results of various stages of deriving a ridge phase representation using the branch cut approach. For simplicity only a central subset of the image, most of which was segmented as foreground, was used for illustration.

Figure 4(f) illustrates that the output cosine of total phase is an acceptable representation over most of the image, but this method suffers from some drawbacks:

- Small inaccuracies in the placement of the branch cuts result in the generation of some spurious features on and near the branch cuts.

- Uncertainties in the orientation estimate in any region traversed by the cut may result in misplacement of later segments of the cut. This problem is not apparent in the example shown here, where the print was of sufficiently high quality to obtain an accurate orientation field over most of the image.

- Branch cuts were easily traced for the simple loop pattern shown here other patterns are not so straightforward, eg. a tented arch pattern contains a core and a delta connected by a single branch cut; twin loop patterns contain spiraling branch cuts which may be very difficult to trace accurately. The model would need modification in order to handle these more difficult cases.

${ }^{3}$ The standard fingertip ridge spacing is about $0.5 \mathrm{~mm}$ (Maltoni, Maio et al. p 83). In our images this corresponds to about 10 pixels. 


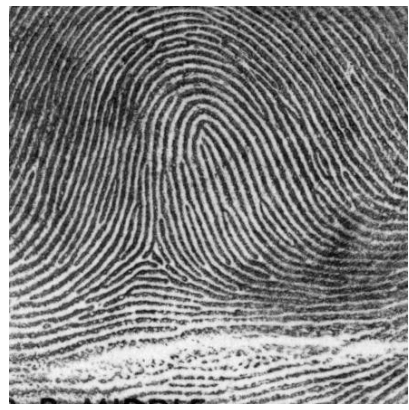

(a) Input image

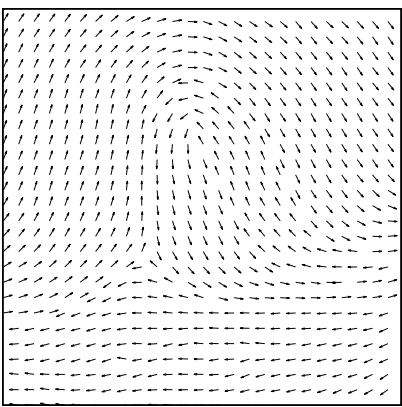

(c) Direction field after disam- ( biguation

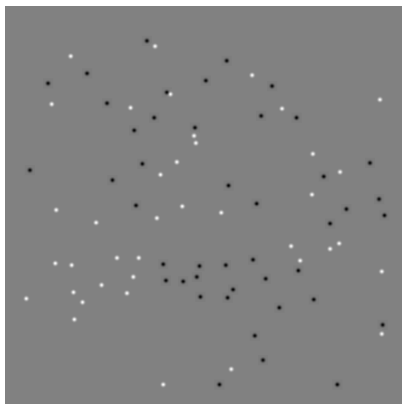

(e) Spiral phase points

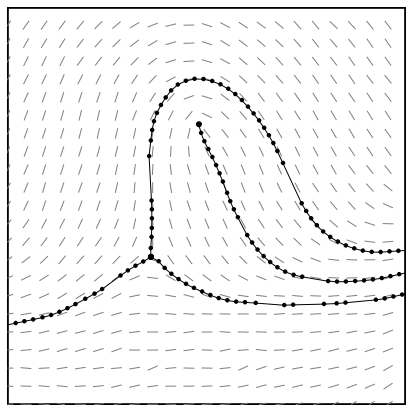

(b) Orientation field, with branch cuts shown

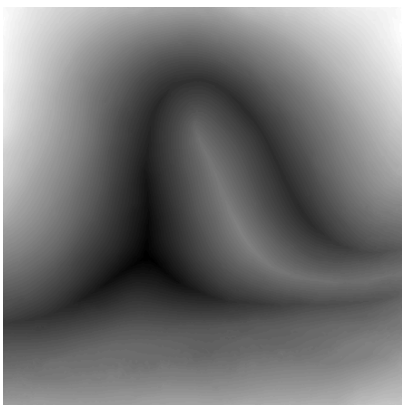

(d) Unwrapped continuous phase

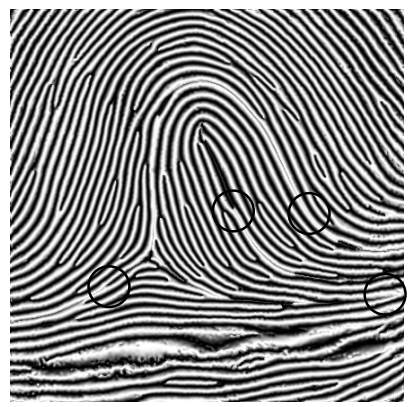

(f) Cosine of total phase.

Fig. 4. Results from disambiguation via branch cuts. White and black dots in (e) represent positive and negative spiral points respectively. Circled regions in (f) indicate where some artifacts appear on and around the branch cuts.

\subsection{Flow Disambiguation via Image Subdivision}

Figure 5 shows the results of flow disambiguation using image subdivision. Because flow direction is not necessarily consistent between neighbouring subimages, the resultant phase sub-images cannot in general be combined into one. 
This drawback is however not too serious, because the value of $\cos (\psi)$ is unaffected when $\psi$ is reversed. In fact we can generate a suitable image of $\cos (\psi)$ from the complete image by applying the demodulation formula using $\beta=\theta+\pi / 2$, where $\theta$ is the orientation, without needing to disambiguate $\theta$. It is only in locating the minutiae that a continuous consistent $\psi$ field is needed, requiring us to perform the demodulation at the sub-image level.

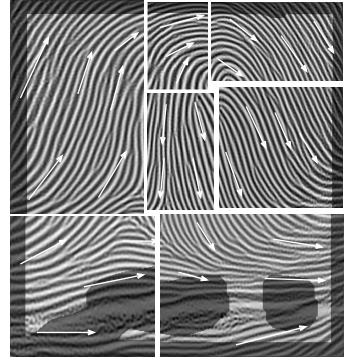

(a) Sample fingerprint image partitioned into subimages.

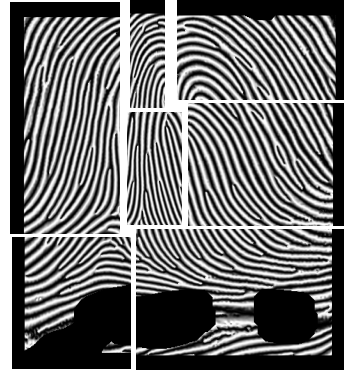

(b) Cosine of ridge phase in each sub-image.

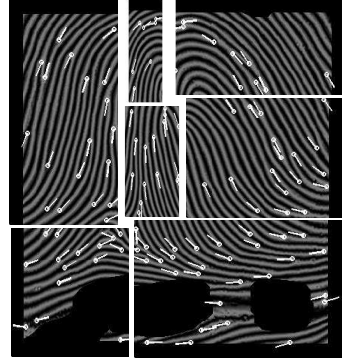

(c) Sub-images with minutiae overlaid.

Fig. 5. Disambiguating the ridge flow by image subdivision. The test image from Fig. 4(a) is subdivided, allowing a consistent flow direction to be assigned for each sub-image (a), although the directions may not be compatible where the sub-images adjoin. Demodulation can then be applied to each sub-image, giving a phase representation of the ridge pattern and allowing the minutiae to be located (c).

\section{Summary}

Two approaches are presented for disambiguating the ridge flow direction - one using branch cuts, and one employing a technique of image subdivision.

The primary advantage of the first method is that it leads to a description of the entire ridge pattern in terms of one continuous carrier phase image, plus a listing of the spiral phase points. The disadvantage is that certain classes of print possess ridge orientation patterns for which it is very difficult or impossible to construct branch cuts, and, even where these can be constructed, certain unwanted artifacts may appear on and near the branch cuts.

The second method does not suffer from these deficiencies. It cannot be used to generate a continuous carrier phase image for the entire pattern - nevertheless we can still obtain a continuous map of the cosine of the phase, and demodulation can be employed on the sub-images to locate the minutiae.

This phase based representation appears to be a more useful way of describing the ridge pattern than a means such as a skeleton ridge map described by chain codes, because the value of the cosine of the phase offers a natural means by which one portion of a fingerprint pattern can be directly compared with another via direct correlation, facilitating fingerprint matching. 
Acknowledgments. The assistance of my supervisors, Dr Peter Kovesi and Dr Du Huynh, in proof-reading the manuscript and contributing many constructive suggestions is gratefully acknowledged. This research was supported by a University Postgraduate award.

\section{References}

Bazen, A.M., Gerez, S.H.: Systematic Methods for the Computation of the Directional Fields and Singular Points of Fingerprints. IEEE Trans. Pattern Analysis and Machine Intelligence 24(7), 905-919 (2002)

Joseph, D.: Helmholtz Decomposition Coupling Rotational to Irrotational Flow of a Viscous Fluid, www.pnas.org/cgi/reprint/103/39/14272.pdf?ck=nck (retrieved May 6, 2008)

Larkin, K.G., Fletcher, P.A.: A Coherent Framework for Fingerprint Analysis: Are Fingerprints Holograms? Optics Express 15(14), 8667-8677 (2007)

Maltoni, M., Maio, D., Jain, A.K., Prabhakar, S.: Handbook of Fingerprint Recognition. Springer, Heidelberg (2003)

Ratha, N.K., Chen, S., Jain, A.K.: Adaptive Flow Orientation-Based Feature Extraction in Fingerprint Images. Pattern Recognition 28(11), 1657-1672 (1995)

Sherlock, B.G., Monro, D.M.: A Model for Interpreting Fingerprint Topology. Pattern Recognition 26(7), 1047-1054 (1993)

Shi, Z., Govindaraju, V.: A Chaincode Based Scheme for Fingerprint Feature Extraction. Pattern Recognition Letters 27, 462-468 (2006) 